\title{
Structure of Mercaptobiphenyl Monolayers on Mercury
}

\author{
Lilach Tamam, Henning Kraack, and Eli Sloutskin \\ Physics Department, Bar-Ilan University, Ramat-Gan 52900, Israel
}

\author{
Benjamin M. Ocko \\ Physics Department, Brookhaven National Laboratory, Upton, New York 11973
}

\section{Peter S. Pershan}

Physics Department, Harvard University, Cambridge, Massachusetts 02138

\author{
Abraham Ulman \\ Chemistry Department, Bar-Ilan University, Ramat-Gan 52900, Israel
}

\author{
Moshe Deutsch* \\ Physics Department, Bar-Ilan University, Ramat-Gan 52900, Israel
}

Received: January 16, 2005; In Final Form: May 5, 2005

\begin{abstract}
The molecular-scale structure and phase behavior of single-component Langmuir films of 4'-methyl-4mercaptobiphenyl (MMB) and 4'-perfluoromethyl-4-mercaptobiphenyl (FMMB) on mercury were studied using surface tensiometry, grazing incidence X-ray diffraction, and X-ray reflectivity. At low coverages, a condensed but in-plane disordered single layer of surface-parallel molecules is found for both compounds. At high coverages, both compounds exhibit in-plane-ordered phases of standing-up molecules. For MMB, the biphenyl core dominates the structure, yielding a centered-rectangular unit cell with an area $A_{\mathrm{x}}$ of $21.8 \AA^{2} /$ molecule, with molecules tilted by $\sim 14^{\circ}$ from the surface normal in the nearest-neighbor direction, and a coherence length $\xi$ of $>1000 \AA$ for the crystalline domains. For FMMB, the perfluoromethyl group dominates the structure, yielding a hexagonal unit cell with untilted molecules, an area $A_{\mathrm{x}}$ of $24.2 \AA^{2} /$ molecule, and a much smaller $\xi$ of $\sim 110 \AA$. The structure is discussed in comparison with self-assembled monolayers of MMB on crystalline $\mathrm{Au}(111)$ and similar-length alkanethiolate SAMs on $\mathrm{Au}(111)$ and on mercury. The differences in the structure are discussed and traced to the differences in the substrate's surface structure, and in the molecular cross section and rigidity.
\end{abstract}

\section{Introduction}

Monolayer and few-layer organic films on liquid and solid surfaces, such as water ${ }^{1}$ and gold, ${ }^{2-4}$ have received ever increasing research attention over the past few decades, because of their importance for both basic and applied sciences. ${ }^{5,6}$ For basic science, these systems allow the study of molecular interactions, structural organization, and rheology in quasi-twodimensional matter. For applied sciences, they provide the means of controlling surface wetting, ${ }^{7}$ chemical and physical patterning of surfaces on micro to nano scales, ${ }^{8}$ two-dimensional (2D) nucleation and growth, ${ }^{9}$ surface-initiated polymerization, ${ }^{10}$ studying electrical transport through the single molecules, ${ }^{11-13}$ and more. Molecular-resolution structural studies of these assemblies on liquids began only two decades ago, with Langmuir films of amphiphilic molecules on water. ${ }^{1,14}$ In these films, the hydrophobic repulsion from the water surface, as well as the intermolecular van der Waals interactions, invariably align the organic molecules roughly normal to the surface, with the hydrophilic headgroup residing in the water and the hydrophobic

* To whom correspondence should be addressed: Physics Department, Bar-Ilan University, Ramat-Gan 52900, Israel. Phone: +972-3-5318476. Fax: +972-3-5353298. E-mail: deutsch@mail.biu.ac.il. tails pointing away from it. The phases and molecular ordering of Langmuir films can be tuned by varying the surface coverage, the temperature, the molecule dimensions, and additives to the subphase. ${ }^{1}$

A different type of monolayer involves the self-assembly of molecules on a solid surface, most notably of thiols on $\mathrm{Au}^{6,15}$ and silanes on $\mathrm{Si}^{16}$ These types of monolayers differ from the previously mentioned Langmuir films for more reasons than just the obvious change of the subphase from a liquid to a solid. The adsorption energies are substantially higher than the $\sim 10$ $\mathrm{kJ} / \mathrm{mol}$ energy of the hydrogen bond of the headgroup to the water subphase in Langmuir films. One- or two-dimensional epitaxy to the ordered, crystalline surface, induced by the strong headgroup-substrate bonding, results in SAMs in a surfaceparallel order longer than that of water-supported Langmuir films, where the liquid surface has only short-range order and the weaker chain-chain van der Waals interaction is the only ordering agent. Note, however, that while for SAMs the order is longer-range, it is dominated by, and thus conforms to, that of the crystalline substrate. The resultant order of the SAM may therefore differ considerably from the order favored by the various intermolecular interactions within the film, which dominate the structure when the subphase is liquid. 
Recently, we have studied in detail Langmuir films of organic monolayers on a new type of substrate: liquid metals. ${ }^{17-20}$ While preserving the strong binding of, for example, thiols to a crystalline Au substrate (a few hundred kilojoules per mole), this substrate has only short-range order so that no external structure is imposed on the monolayer. Moreover, the liquid surface comprises mobile molecules, and allows the same type a continuous increase or decrease of the surface pressure and surface coverage by the monolayer as for conventional Langmuir films on water. An additional advantage of such liquid metal subphases is their high surface tension, $\sim 490 \mathrm{mN} / \mathrm{m}$ for the mercury subphase used in this study. This, in turn, provides a supersmooth substrate (with roughness amplitudes of only $\sim 1.5$ $\AA$; see below) of macroscopic lateral dimensions, which allows the X-ray reflectivity method employed here to reach resolutions of $\leq 1 \AA$, which is $\sim 4$ times better than those which can be achieved on a water subphase. Moreover, mercury is emerging as a soft metallic contact of choice for charge-transfer studies through single molecules in the field of molecular electronics. ${ }^{12,13}$ This attracts additional interest in the structure of mercury-supported organic monolayers for molecules having favorable electronic properties for device construction, such as the conjugated aromatics studied here.

Over the past few years, SAMs of conjugated aromatic thiols on crystalline $\mathrm{Au}$ surfaces became the focus of increasing research interest. Originally, this was due to an interest in the influence of the backbone rigidity on the structure. ${ }^{2}$ This led to the study of the MMB molecule, which has the same length as the best-studied alkyl-thiol, $n$-decanethiol, but consists of the much more rigid biphenyl unit. An additional reason for the interest in conjugated aromatics is their relative abundance of delocalized electrons, ${ }^{3,21}$ which implies a higher electrical conduction through, and across, the molecular backbone, a desirable property for molecular electronic devices.,22 In comparison with $n$-alkyl-thiols, the aromatic molecules are highly anisotropic, and their intermolecular interactions are more defined. ${ }^{3}$ This potential for generating a better- and differentordered in-plane molecular packing, as compared to that of alkyl-thiols, is demonstrated by the structure of MMB SAMs on $\mathrm{Au}^{2}{ }^{2}$ Two phases of varying density were observed, a lowdensity surface-parallel, or lying-down, phase and a high-density surface-normal, or standing-up, phase. The lying-down phase exhibited a diffraction pattern consistent with an Au-surfacecommensurate rectangular $(8 \times 2 \sqrt{ } 3)$ surface lattice with four molecules having a head-to-head molecular orientation, while the standing-up phase showed a diffraction pattern consistent, again, with an Au-commensurate hexagonal $(\sqrt{ } 3 \times \sqrt{ } 3)$ R30 lattice with a molecular tilt of less than $19^{\circ}$ from the surface normal. ${ }^{2}$ The phases of alkyl-thiolates on $\mathrm{Au}$, both $\mathrm{CH}_{3}$ - and $\mathrm{CF}_{3}$-terminated, are considerably different, in both their lyingdown and standing-up phases. ${ }^{23}$

We present here a study of Langmuir films of biphenyl thiols on mercury. We used surface tension isotherms and surfacespecific X-ray measurements (grazing incidence diffraction and reflectivity) to characterize the thermodynamics and structure of the monolayer, respectively. Two biphenyl thiols were used, a protonated 4'-methyl-4-mercaptobiphenyl $\left(\mathrm{CH}_{3}-\mathrm{C}_{6} \mathrm{H}_{4}-\mathrm{C}_{6} \mathrm{H}_{4}\right.$ $\mathrm{SH}$ ), denoted $\mathrm{MMB}$, and a trifluoro analogue, $4^{\prime}$-trifluoromethyl4-mercaptobiphenyl $\left(\mathrm{CF}_{3}-\mathrm{C}_{6} \mathrm{H}_{4}-\mathrm{C}_{6} \mathrm{H}_{4}-\mathrm{SH}\right)$, denoted FMMB. The trifluoromethyl functionality, which creates a large molecular dipole moment in FMMB, not existing in $\mathrm{MMB},{ }^{24}$ and increases the end group's cross section, should provide an additional "knob", beyond the surface pressure, for changing the structure of the monolayer in its various phases.
SCHEME 1: MMB (left) and FMMB (right) ${ }^{a}$

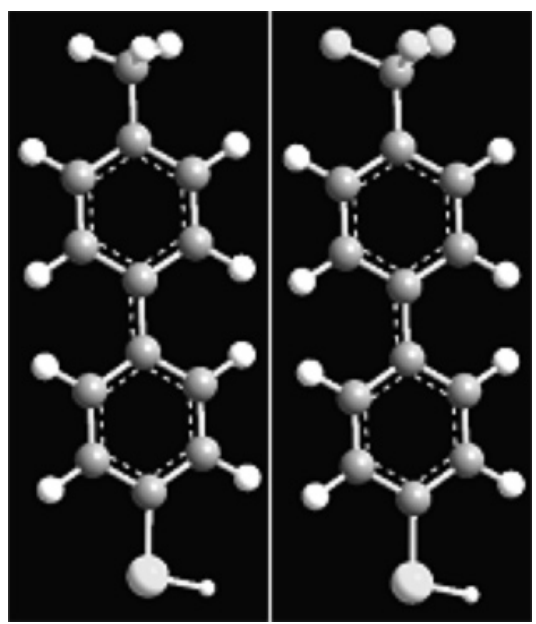

${ }^{a}$ The SH groups are at the bottom, and the protonated (MMB) and perfluorinated (FMMB) methyl groups are at the top.

\section{Experimental Section}

The surface thermodynamics were studied by surface pressure $(\pi)$ - molecular area $(A)$ isotherms. The structure of the Langmuir film was studied by surface-specific X-ray methods using synchrotron radiation. Both types of measurements were carried out in a Langmuir trough, which could be mounted on the X-ray diffractometer for in-situ X-ray measurements. The experimental and data analysis methods, particularly for the $\mathrm{X}$-ray measurements, have been described previously ${ }^{17-19}$ and thus will be discussed here only briefly.

Trough. A specially designed Langmuir trough, with an area of $6.5 \mathrm{~cm} \times 17.5 \mathrm{~cm}$, made of KelF and enclosed in a hermetically sealed box was used in the measurements. The box had Kapton windows to allow X-ray measurements, and was filled with helium (X-ray measurements) or nitrogen (isotherm measurements) to reduce the level of both surface contamination of the mercury and beam damage during X-ray measurements. During X-ray measurements, the trough was supported on an active vibration isolation unit, mounted on the X-ray diffractometer. This arrangement was shown in our previous measurements ${ }^{17,20,25,26}$ to effectively eliminate the surface fluctuations arising from environmental vibrations that are a major problem in this type of X-ray measurement.

The surface tension was measured by the Wilhelmy plate method, ${ }^{27}$ using an $\mathrm{Hg}$-amalgamated platinum plate, and a homemade balance based on a linear variable differential transformer (LVDT). The molecular area $A$ was varied by stepwise deposition of metered volumes of chloroform-dissolved samples. The trough's temperature was controlled to $\pm 0.2^{\circ} \mathrm{C}$ by a commercial water circulator. Room-temperature measurements were carried out at $25^{\circ} \mathrm{C}$.

Materials. Mercury was purchased from Merck (tripledistilled, 99.999\% pure) or from Bethlehem Apparatus (quadrupledistilled, 99.9995\% pure). The two yield isotherms which are indistinguishable within our experimental accuracy.

Two molecules, shown in Scheme 1, were investigated in this study: 4'-methyl-4-mercaptobiphenyl, $\mathrm{CH}_{3}-\mathrm{C}_{6} \mathrm{H}_{4}-\mathrm{C}_{6} \mathrm{H}_{4}-\mathrm{SH}$ (denoted $\mathrm{MMB}$ ), and a trifluoromethyl analogue, $4^{\prime}$-trifluoromethyl-4-mercaptobiphenyl, $\quad \mathrm{CF}_{3}-\mathrm{C}_{6} \mathrm{H}_{4}-\mathrm{C}_{6} \mathrm{H}_{4}-\mathrm{SH}$ (denoted FMMB). The synthesis of the materials is described elsewhere. ${ }^{28}$

Standard spreading solutions were prepared with molarities in the range of $3-8 \times 10^{-4}$ using HPLC grade, $99.9 \%$ pure chloroform (Aldrich). 


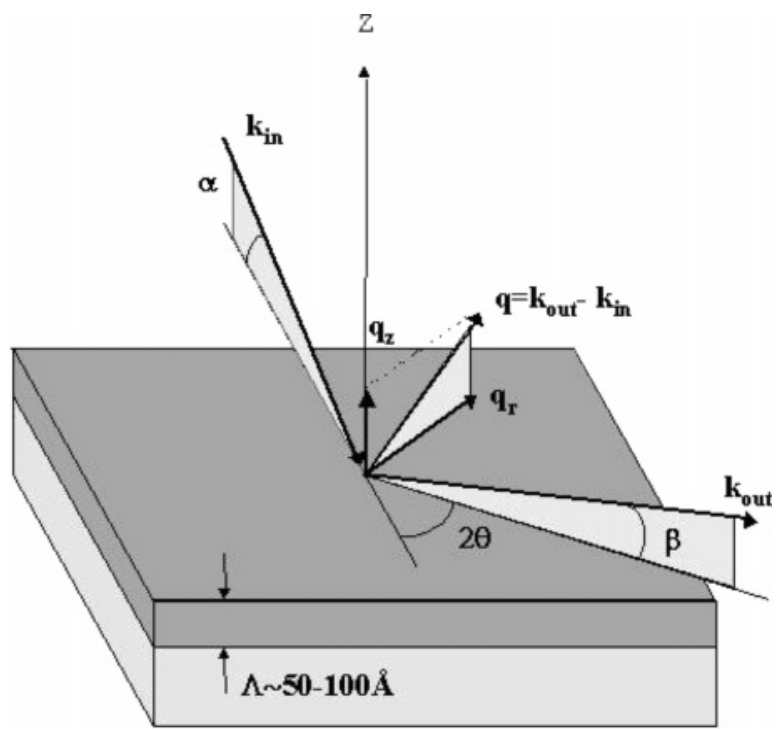

Figure 1. Diagram of the surface-specific X-ray diffraction geometry. For a discussion, see the text.

Isotherm Measurements. The surface thermodynamics were studied by surface pressure $(\pi)$-molecular area $(A)$ isotherm measurements. Here $\pi=\gamma_{\mathrm{Hg}}-\gamma$, the difference in surface tension between the bare $\left(\gamma_{\mathrm{Hg}} \approx 490 \mathrm{mN} / \mathrm{m}\right)$ and the filmcovered $(\gamma)$ surface. $A$ is the nominal area per molecule as calculated from the number of molecules deposited on the surface and the trough area. For the in-plane-ordered phases of the film, an accurate value for $A$ can be derived from the unit cell dimensions determined from the X-ray measurements. This molecular area is denoted in the following by $A_{\mathrm{x}}$.

As a well-sealing movable barrier is difficult to construct for a mercury subphase, ${ }^{29}$ the molecular area $A$ was reduced by stepwise deposition of additional solution onto the surface, rather than by the conventional method of barrier compression. Each step consisted of 3-5 $\mu \mathrm{L}$, deposited using an adjustable microsyringe. This is equivalent to an increase of $5-25 \times 10^{-4}$ molecules/ $\AA^{2}$ per step (depending on the concentration of the solution) in the areal density of the molecules. The surface tension variation following each deposition was monitored, and the next step was carried out only after $\gamma$ has been observed by eye to relax to a roughly constant value. These relaxation times varied from $\sim 30 \mathrm{~s}$ at low areal densities to $\sim 250 \mathrm{~s}$ at high areal densities.

X-ray Measurements. The structure of the Langmuir films was studied in situ on the trough with subangström resolution using surface-specific X-ray techniques. The X-ray measurements were carried out at the Harvard BNL Liquid Surface Diffractometer at National Synchrotron Light Source (NSLS) at beamline $\mathrm{X} 22 \mathrm{~B}$, with $\mathrm{X}$-ray wavelengths $\lambda=1.539$ and 1.523 $\AA$.

The geometry used for the X-ray measurements is shown in Figure 1. The incident rays have a wavevector $\mathbf{k}_{\text {in }}$. The detector selects a beam with a diffracted wavevector $\mathbf{k}_{\text {out }}$ according to its spatial position, and integrates the intensity over its angular acceptance range (i.e., its angular resolution). The momentum transfer, $\mathbf{q}$, is then given by $\mathbf{q}=\mathbf{k}_{\text {out }}-\mathbf{k}_{\text {in }}$, where $\left|k_{\text {in }}\right|=\left|k_{\text {out }}\right|$ $=2 \pi / \lambda$. The surface-normal, $q_{\mathrm{z}}$, and surface-parallel, $q_{\mathrm{r}}$, components of $\mathbf{q}$ are

$$
q_{\mathrm{z}}=k_{\mathrm{in}}(\sin \alpha+\sin \beta)
$$

$$
q_{\mathrm{r}}=k_{\text {in }} \sqrt{\cos ^{2} \alpha+\cos ^{2} \beta-2 \cos \alpha \cos \beta \cos 2 \theta}
$$

(1) X-ray reflectivity (XR) measurements, ${ }^{30}$ where the ratio of intensities of the incident and specularly reflected rays, $R\left(q_{z}\right)$, is measured. This is done by varying the incidence angle under the conditions that $\alpha=\beta$ and $\theta=0$. In this geometry, the wave vector transfer is normal to the surface: $q_{\mathrm{z}}=(4 \pi / \lambda) \sin$ $\alpha \neq 0$ and $q_{\mathrm{r}}=0 . R\left(q_{\mathrm{z}}\right)$ provides information about the surfacenormal structure of the Langmuir film, i.e., its surface-normal electron density profile, its layer thickness, and its surface roughness. ${ }^{31}$

(2) Grazing incidence diffraction (GID), which measures the intensity diffracted at a surface-parallel angle $2 \theta$ away from the incidence plane for a fixed incidence angle $\alpha<\alpha_{c}$, where $\alpha_{c}$ is the critical angle for total external reflection of X-rays. ${ }^{31}$ In this case $q_{\mathrm{r}} \neq 0$, and the detected intensity is integrated over the surface-normal $q_{\mathrm{z}}$ acceptance range of the detector. GID provides information about the surface-parallel structure of the film. Crystalline order will produce Bragg diffraction peaks at $q_{\mathrm{r}}($ or $2 \theta$ ) positions just like in conventional crystallography of three-dimensional (3D) crystals.

(3) Bragg rods (BR), which are the surface-normal (i.e., $q_{\mathrm{z}}$ ) distributions of the diffracted intensity at the peak positions in the GID pattern. In that case, $q_{\mathrm{r}} \neq 0$ is fixed and $q_{\mathrm{z}}$ is scanned. BR provide information about the molecular tilt and its azimuthal direction, and the length of the part of the molecule which gives rise to the particular BR.

The XR was measured in our case by a point detector (i.e., an NaI scintillation detector having a single rectangular slit in front of it), and the GID and BR are measured simultaneously using a linear position sensitive detector (PSD), aligned along the surface normal. A Soller slit, positioned in front of the PSD, provided a horizontal resolution $\Delta q_{\mathrm{r}}$ of $0.017 \AA^{-1}$. For a GID scan, a full $q_{\mathrm{z}}$-resolved rod is measured at each $q_{\mathrm{r}}$ position, and this allows the construction of an equal-intensity contour plot that describes the diffracted intensity landscape in the $\left(q_{\mathrm{r}}, q_{\mathrm{z}}\right)$ plane. This provides an excellent overview of the film's structure, and allows easy differentiation of features originating in the qausi-2D film from those that originate in the few small 3D crystallites that may form within the film, particularly at high surface coverages that are close to film collapse.

To minimize beam damage, an automatic shutter was employed to block the beam at all times other than the actual counting period at each point. This, and periodic translations of the trough in the beam-normal direction, particularly during the longer, higher-flux, GID measurements, provided reproducible results free from beam-generated damage to within the accuracy of our measurements.

\section{Results}

Pressure-Area Isotherms. The $\pi-A$ isotherms of MMB and FMMB on mercury, measured at $25^{\circ} \mathrm{C}$, are shown in Figure 2. They are qualitatively similar. As $A$ is reduced from $\sim 300$ $\AA^{2} /$ molecule, both show (a) a flat, near-zero region for $A>$ $100 \AA^{2} /$ molecule, (b) a fast rise to $\pi \approx 30-40 \mathrm{mN} / \mathrm{m}$ at $A \approx$ $70 \AA^{2} /$ molecule, (c) a plateau at $\pi \approx 30-40 \mathrm{mN} / \mathrm{m}$ down to $A$ $\approx 35-40 \AA^{2} /$ molecule, and finally (d) a fast rise below this $A$ to film collapse at $A \approx 20 \AA^{2} / \mathrm{molecule}$ and $\pi \approx 60-80 \mathrm{mN} / \mathrm{m}$. On the basis of our recent measurements on Langmuir films of fatty acids, ${ }^{17}$ alkanes, ${ }^{18}$ alcohols, ${ }^{19}$ and alkyl-thiols ${ }^{20}$ on mercury, and on the basis of the X-ray results discussed below, these four regions can be identified as (a) a 2D gas of lying-down molecules, (b) a single layer of condensed lying-down molecules, (c) a coexistence of lying-down and standing-up molecules, and (d) a monolayer of standing-up molecules. We now discuss these regions in some detail. 


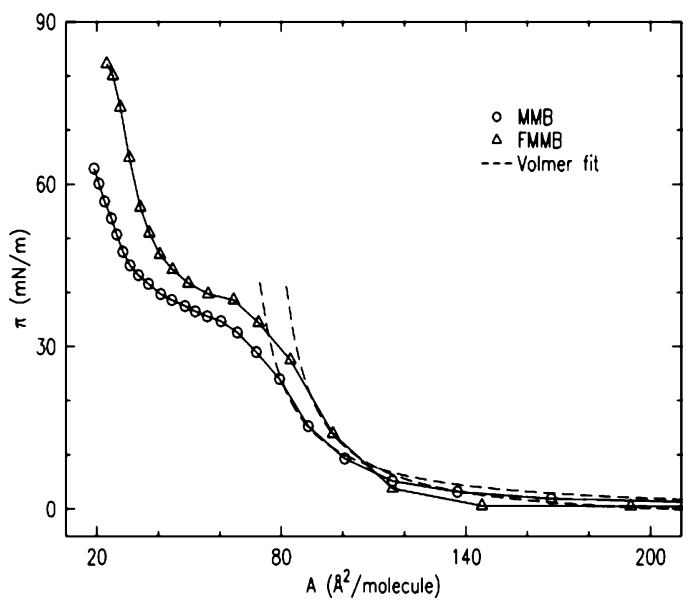

Figure 2. Surface pressure $(\pi)-$ molecular area $(A)$ isotherms of MMB and FMMB on mercury. A fit to the Vollmer equation, the isotherm of a gas of hard disks (dashed lines), yields exclusion areas $A_{0}=63.5$ $\AA^{2} /$ molecule for MMB and $A_{0}=73 \AA^{2} /$ molecule for FMMB.

The reduction of the molecular area $A$ from $\sim 220$ to $\sim 100$ $\AA^{2} /$ molecule results in only a few millinewtons per meter increase in the surface pressure, as expected for a dilute 2D gas. Reducing $A$ to below $100 \AA^{2} /$ molecule results in a very steep rise in the surface pressure from $\pi \approx 6 \mathrm{mN} / \mathrm{m}$ at $\sim 100$ $\AA^{2} / \mathrm{molecule}$ to $\pi \approx 35 \mathrm{mN} / \mathrm{m}$ at $\sim 65 \AA^{2} / \mathrm{molecule}$. These fastincreasing parts of the isotherms are very well fitted by the Vollmer equation for a $2 \mathrm{D}$ gas of hard spheres $\pi\left(A-A_{0}\right)=$ $k_{\mathrm{B}} T$, where $k_{\mathrm{B}}$ is the Boltzmann constant and $A_{0}$ is the exclusion area due to the finite size of the sphere. The fits are shown as dashed lines in Figure 2 and yields exclusion areas $A_{0}=63.5$ $\AA^{2} /$ molecule for MMB and $A_{0}=73 \AA^{2} /$ molecule for FMMB.

The $A_{0}$ value of MMB is very close to the calculated area of the molecule, $65 \AA^{2}$, obtained from the $13.4 \AA$ calculated length of the molecule 2,32 (see below), and the $4.8 \AA$ average between the widths of lying-down (6.3 $\AA$ ) and standing-up (3.3 $\AA$ ) phenyl rings. This average is in line with the $90^{\circ}$-rotated conformation of the two phenyls, found here (see below) and on $\mathrm{Au}(111) . A_{0}$ of MMB is also close to the value of $57.7 \AA^{2} /$ molecule found by Leung et al. ${ }^{2}$ for the single striped phase observed in their $\mathrm{X}$-ray study of vapor-deposited $\mathrm{MMB}$ on $\mathrm{Au}(111){ }^{2}$ In this phase, the molecular long axis is aligned parallel to the surface, with one phenyl ring lying flat on the surface, and the other rotated (around the molecular long axis) at an angle away from it. Although this phase was not found in the STM study of liquid-adsorbed $\mathrm{MMB}$ on $\mathrm{Au}(111)$ by Azzam et al., ${ }^{32}$ their striped $\beta$ phase yields $72 \AA^{2} /$ molecule, not too far from the present $A_{0}$. In this phase, the molecules are found to lie flat on the surface. The coincidence of $A_{0}$ with these values and with the calculated area of the molecule implies therefore that the rise in the isotherm near $A_{0}$ is due to the close packing of a monolayer of flat-lying molecules for both MMB and FMMB. This conclusion is supported by the X-ray measurements detailed below.

The onset of the plateau at $A \approx 60 \AA^{2} /$ molecule for MMB and $A \approx 70 \AA^{2} /$ molecule for FMMB marks the completion of a densely packed single layer (SL) of lying-down molecules, and the onset of the coexistence range between standing-up and lying-down phases. With further decreases in $A$, increasingly more of the molecules stand up, until a pure standing-up phase is obtained at the onset of the steep rise at $A$ values of $A \approx 30$ $\AA^{2} /$ molecule for MMB and $A \approx 40 \AA^{2} /$ molecule for FMMB. The steep rise indicates low compressibility, as expected from the condensed solid 2D phase at these low areas per molecule, which are close to those found for the standing-up phases of fatty
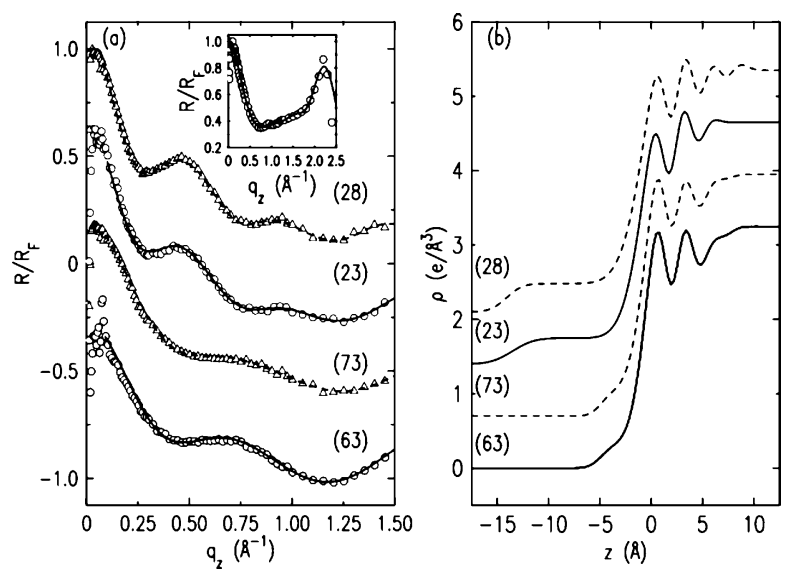

Figure 3. (a) Measured Fresnel-normalized X-ray reflectivity curves of MMB $(O)$ and FMMB $(\triangle)$ on mercury, with box model fits $(-)$. Curves are shifted vertically by 0.4 each for clarity. The inset shows the same for a bare $\mathrm{Hg}$ surface. (b) Surface-normal electron density profiles derived from the fits in panel a. MMB and FMMB results are shown as solid and dashed lines, respectively. The $\mathrm{Hg}$ surface is at $z$ $=0$, with $z>0$ pointing into the liquid. Curves are shifted by 0.7 for clarity. Numbers in parentheses are the nominal molecular areas in $\AA^{2} /$ molecule.

acids $^{17}$ and alcohols ${ }^{19}$ on mercury and on water. ${ }^{1}$ The X-ray measurements discussed below support the inferences in these paragraphs, and show that long-range order indeed exists in the pure standing-up phases of both molecules.

Finally, we note that very high surface pressures can be achieved in these monolayers on mercury, before the pressure causes the film to collapse. The values found here, and in particular that of FMMB, $80 \mathrm{mN} / \mathrm{m}$, are $\sim 20 \mathrm{mN} / \mathrm{m}$ higher than even those found for alkanethiols on mercury ${ }^{20}$ and $\sim 40 \mathrm{mN} / \mathrm{m}$ higher than those found for Langmuir films on aqueous subphases. ${ }^{1}$ This is partly due to the strong attraction of the film to the mercury surface and partly to the strong chemical bond, of a few hundred kilojoules per mole, between the sulfur of the headgroup and the underlying mercury, none of which exist on aqueous subphases. The area per molecule observed in the isotherms at collapse, $\sim 20 \AA^{2} /$ molecule, is consistent with our X-ray results below, the value of $\sim 22 \AA^{2} /$ molecule measured by X-rays by Leung et al. ${ }^{2}$ for the hexagonal standing-up phase, and the same value measured using STM by Azzam et al. ${ }^{32}$ for the $\chi$ and $\epsilon$ phases for MMB on $\mathrm{Au}(111)$.

To confirm the conclusions derived from the isotherms, and detect possible lateral order within the film, we have carried out surface-specific X-ray measurements, which will be described in the next section.

X-ray Reflectivity. Figure 3 a shows a selection of the Fresnel-normalized XR curves (circles) measured at the indicated coverages, along with their model fits (lines), for MMB (solid line) and FMMB (dashed lines). The XR curves shown, two for each of the two molecules, were measured at the onset of the plateau and in the low- $A$, fast-rising regions of each of the two isotherms shown in Figure 2.

A rough estimate of the structure in the surface-normal direction can be obtained from the period of the modulations, $\Delta q_{\mathrm{z}}$, in these curves, which are related to the layer thicknesses, $D$, of the corresponding films by the equation $\Delta q_{\mathrm{z}}=2 \pi / D$. The amplitude of the modulations depends on the electron density differences among the gas phase, the surface layer, and the bulk and also on the interfacial roughness. ${ }^{33}$ For the two low densities, 63 (MMB) and $73 \AA^{2} /$ molecule (FMMB), $\Delta q_{z}$ $\approx 0.9 \AA^{-1}$, which yields a $D \approx 6.9 \AA$. This value is considerably less than the calculated length of the molecule, (13.4 
$\AA$ ), but is not too far from the $6.3 \AA$ diameter of the phenyl ring, which is the width of the molecule. This implies that at this coverage the molecules do not stand up and their long axis should be parallel to the surface, or nearly so. For the high densities, 23 (MMB) and $28 \AA^{2} /$ molecule (FMMB), $\Delta q_{\mathrm{z}} \approx 0.47$ $\AA^{-1}$, which yields a $D \approx 13.4 \AA$. This value corresponds well to the expected molecular length, $13.4 \AA$, obtained when adding to the $10.4 \AA$ "S-to-methyl-C" length of the molecule ${ }^{2,32}$ the $\mathrm{S}-\mathrm{Hg}$ bond length of approximately ${ }^{34} 2.5 \AA$ and the $0.5 \AA$ "methyl-C-to-topmost-H" bond length, ${ }^{35}$ projected onto the molecular axis. The correspondence of these values indicates that at these coverages the molecules are oriented with their long axis roughly normal to the surface. These estimated values confirm, therefore, the conclusions derived above from the isotherms that the low-coverage and high-coverage phases comprise lying-down and standing-up molecules, respectively. These estimates are, however, rather rough, particularly for the lying-down phase where the modulation period is difficult to determine since only two minima are observed in the XR curves. A more accurate determination of the structure requires detailed modeling. The best fits by the model constructed as detailed below are shown in Figure $3 \mathrm{a}$ in lines, with the corresponding surface-normal density profiles plotted in Figure 3b. These fits confirm the conclusions derived here and provide additional details of the structure.

The box model employed here to describe the surface-normal electron density profile is identical with that used in previous studies. ${ }^{17-20}$ It consists of eight "boxes": a single box of constant electron density for each molecular overlayer (a single layer in this study) and seven boxes to represent the surfacelayered mercury subphase. Each box has a width, a constant electron density, and a roughness at its interfaces with the adjacent boxes. For the overlayer box, the electron density was calculated by dividing the number of electrons in the molecule by the molecular volume. For the standing-up phases, the X-ray GID measurements provide accurate dimensions for the unit cell, and thus, the molecular volume can be calculated accurately. For the disordered lying-down phase, where no GID peaks are found, this volume was calculated from the dimensions of the molecules, and slight variations were allowed so that good fits could be obtained. The basic features of the model for the $\mathrm{Hg}$ subphase were determined from our previous studies of Langmuir films on mercury. ${ }^{17-20}$ The width of the topmost box (representing the thickness of the film) was varied in the fit, either as a free fit parameter or, when strongly correlated with other fit parameters, as a fixed, but changing, value in an iterative series of fits. The widths of the seven boxes representing the layered density profile of the mercury subphase were fixed at $1.3 \AA$, half the atomic layer spacing at the mercury surface. ${ }^{20,25}$ All interfacial roughness values between these boxes were taken to be constant at $0.7 \AA$. The electron densities of the boxes were varied, except for that of the first box, which was fixed at $5.5 \pm 0.2 \mathrm{e} / \AA^{3}$, an average of numerous trial fits for different reflectivity curves. The mercury's bulk electron density (the last box) was also kept fixed at the value of 3.25 e/ $\AA^{3}$ calculated from the known mass density of mercury at room temperature. These procedures were found in previous studies of Langmuir films on mercury ${ }^{17-20}$ to yield very good fits, and consistent, physically reasonable results. They were, therefore, adopted here as well. As can be observed in Figure 3, this model provides excellent fits to the present data as well, over a broad $q_{\mathrm{z}}$ range and 8-9 orders of magnitude in the reflectivity, $R\left(q_{\mathrm{z}}\right)$.

The density profiles derived from the fits are shown in Figure $3 \mathrm{~b}$, with $z=0$ taken at the position of the mercury-film
TABLE 1: Nominal Coverages, $A$, Calculated from the Amount of Material Deposited, Layer Thicknesses, $d$, and Roughnesses of the Molecule-Air $\left(\sigma_{\text {molecule }}\right)$ and Mercury-Molecule $\left(\sigma_{\mathrm{Hg}}\right)$ Interfaces $^{a}$

\begin{tabular}{lcrccc}
\hline molecule & $A\left(\AA^{2}\right)$ & \multicolumn{1}{c}{$d(\AA)$} & phase & $\sigma_{\text {molecule }}(\AA)$ & $\sigma_{\mathrm{Hg}}(\AA)$ \\
\hline MMB & 63 & $4.9(1.2)$ & SL & $1.2(0.5)$ & $1.3(0.5)$ \\
& 23 & $13.5(0.5)$ & ML & $1.7(0.3)$ & $1.9(0.3)$ \\
FMMB & 73 & $4.9(1.2)$ & SL & $0.9(0.5)$ & $1.6(0.5)$ \\
& 28 & $14.1(0.5)$ & ML & $1.2(0.3)$ & $2.1(0.3)$
\end{tabular}

${ }^{a} \mathrm{SL}$ represents the single-layer phase of lying-down molecules and ML the monolayer of standing-up molecules. Numbers in parentheses are estimated uncertainties.

interface, and the positive $z$-axis pointing into the subphase. The previously detected ${ }^{25}$ surface layering peak of mercury is observed at $q_{\mathrm{z}} \approx 2.2 \AA^{-1}$ in the bare mercury reflectivity curve in the inset of Figure 3a. The fit results are summarized in Table 1. The fits yield a surface roughness value in the range of 1.3 $\pm 0.5 \AA$. A slightly larger value, $1.7 \pm 0.5 \AA$, is obtained for the film-mercury interface. Both are close to the roughness of the bare mercury free surface. ${ }^{25}$

The MMB fit at $A=63 \AA^{2} /$ molecule, the onset of the plateau, yields a layer thickness $d$ of $4.9 \AA$ and an electron density $\rho_{\mathrm{e}}$ of $0.3 \mathrm{e} / \AA^{3}$. This $d$ value agrees very well with the average of the diameter $(6.3 \AA)$ and thickness $(3.3 \AA)$ of the phenyl ring, ${ }^{2}$ discussed above, suggesting that one phenyl ring lies flat while the other is rotated by $\sim 90^{\circ}$ and stands on its side. This molecular conformation is found also for the stripe phase of MMB on $\mathrm{Au},{ }^{2}$ where the molecular area is somewhat smaller (57.7 $\AA^{2} /$ molecule). This conclusion is valid also for FMMB, for the same reasons. Note, however, that the small thickness of this layer, which results in only a single Kiessig fringe being measurable in the reflectivity curve, renders the uncertainty in this value relatively large. The situation is much improved in the standing-up phase, where more fringes could be measured.

The MMB fit at $A=23 \AA^{2} /$ molecule yields a uniform film with a thickness $d$ of $13.5 \AA$ and an electron density $\rho$ of 0.35 $\mathrm{e} / \AA^{3}$. This $d$ agrees well with the extended "terminal-H-to-Hg" length of a surface-normal aligned molecule of MMB, indicating that at this coverage the MMB molecules are aligned along the surface normal. However, since the apparent film thickness derived from the XR measurements has a cosine dependence on the molecular tilt, a small tilt from the vertical cannot be excluded. Indeed, the GID measurements discussed below reveal such a tilt of $\sim 14^{\circ}$. This changes the model thickness by only $0.4 \AA$, which is within the error bar of the XR model fits.

The electron density obtained from the fit, $\rho_{\mathrm{e}}=0.35 \mathrm{e} / \AA^{3}$ coincides with the calculated $\rho_{\mathrm{e}}($ calcd $)=104 /(21.9 \times 13.5)$ $=0.35 \mathrm{e} / \AA^{3}$, where we used the molecular area $A_{\mathrm{x}}=21.9 \AA^{2} /$ molecule, obtained below from the GID measurements for this ordered phase, the length $d=13.5 \AA$ obtained here from the XR measurements (see Table 1), and the 104 electrons of the molecule.

For FMMB at $A=28 \AA^{2} /$ molecule the XR fit yields a thickness $d=14.1 \AA$ and an electron density $\rho_{\mathrm{e}}=0.38 \mathrm{e} / \AA^{3}$, which agrees well with a calculated length of $14.2 \AA$. This length includes the $\mathrm{S}-\mathrm{Hg}$ bond length ${ }^{34}, 2.5 \AA$, the S-to-methyl-C distance of ${ }^{2,32} 10.4 \AA$, and an axis-projected length of ${ }^{36} 1.3 \AA$ for the "methyl-C-to-topmost-F" distance plus the van der Waals radius of the topmost $\mathrm{F}$. The value of the electron density, $\rho_{\mathrm{e}}=$ $0.38 \mathrm{e} / \AA^{3}$, agrees with that calculated from $d=14.2 \AA$, the molecular area $A_{\mathrm{x}}=24.2 \AA^{2}$ derived below from the GID measurements, and the 130 electrons of the molecule, yielding $\rho_{\mathrm{e}}($ calcd $)=130 /(24.2 \times 14.2)=0.38 \mathrm{e} / \AA^{3}$.

Grazing Incidence Diffraction. The GID measurements reveal no in-plane order in the low-coverage, lying-down phases 

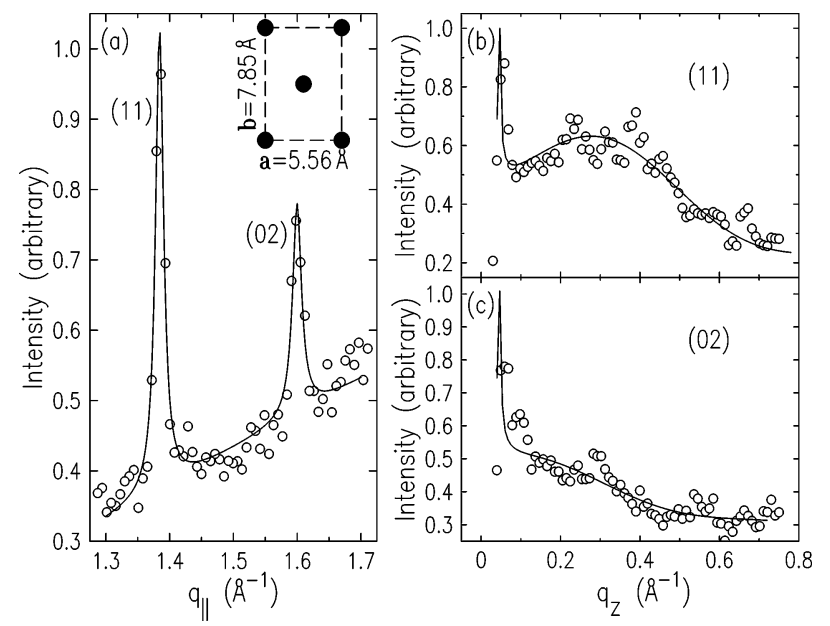

Figure 4. Measured $(O)$ and model-fitted $(-)$ GID (a) and BR (b and c) for the standing-up phase of MMB at room temperature. The inset of panel a shows the body-centered rectangular unit cell derived from the GID pattern in panel a.

of either the MMB or the FMMB. For the high-coverage, standing-up phases, different order is found for MMB, which exhibits a body-centered 2D unit cell, and FMMB, which exhibits a weak 2D hexagonal packing. We now discuss these results separately for the two materials.

$M M B$. The GID scan for MMB spanned the range of $0.3<$ $q_{\|}<1.73 \AA^{-1}$. As shown in Figure 4a, the high coverage, standing-up phase of MMB at a nominal $A=30 \AA^{2} /$ molecule and $T=25{ }^{\circ} \mathrm{C}$ exhibits two GID peaks: $q_{\|}=1.384 \AA^{-1}$ and $q_{\|}=1.600 \AA^{-1}$. The $q_{\|}=1.384 \AA^{-1}$ peak appears immediately after deposition of a new film on the mercury surface. However, the $q_{\|}=1.600 \AA^{-1}$ peak was only observed $\sim 5 \mathrm{~h}$ after the film deposition. No further changes were observed in the GID pattern after this period. A single GID peak indicates a hexagonal phase, which can be represented alternatively by a body-centered rectangular unit cell with dimensions of $5.24 \AA$ $\times 5.24 \sqrt{ } 3 \AA$ with two molecules per unit cell. This yields $A_{\mathrm{x}}$ $=23.8 \AA^{2} /$ molecule, which is $\sim 10 \%$ larger than the molecular area of the same molecule on $\mathrm{Au}(111)$, at the same nominal coverage. ${ }^{2}$

On the other hand, the two peaks observed $5 \mathrm{~h}$ after deposition can only be indexed in terms of a body-centered rectangular unit cell that is shown in the inset of Figure 4a. The two GID peaks are the lowest-order nonvanishing [i.e., even $-(h+k)$ ] peaks, (11) and (02), in a body-centered 2D rectangular cell with dimensions of $5.56 \AA \times 7.85 \AA$ with two molecules per unit cell, i.e., $A_{\mathrm{x}}=21.8 \AA^{2} /$ molecule. While it is possible to assign the time-dependent change observed in the GID pattern to a structural transition from a hexagonal to a rectangular cell, it is more likely that only one phase is observed at all times, and the absence of the $q_{\|}=1.600 \AA^{-1}$ peak is due to the selforganization time of the crystalline domains. This is further supported by the fact that the single BR that was measured for the (11) peak $\sim 2 \mathrm{~h}$ after deposition, when the (02) peak was still not observed, is very similar, albeit within a large scatter due to its low intensity, to that shown in Figure 4b: it peaks at $q_{\mathrm{z}} \approx 0.3 \AA^{-1}$. For a hexagonal packing, surface-normal molecules, showing a BR peaking at $q_{\mathrm{z}}=0 \AA^{-1}$, similar to that in Figure $4 \mathrm{c}$, would have been expected. Note also that the (11) peak at $1.384 \AA^{-1}$ is 4 times degenerate while the (02) peak at $1.600 \AA^{-1}$ is only doubly degenerate. Thus, at the early stages of the self-organization of the crystalline domains, when the domains are still small and thus scatter weakly, the stronger (11) $q_{\|}=1.384 \AA^{-1}$ peak is observed, while the weaker (02) $q_{\|}=1.600 \AA^{-1}$ peak is still too weak to observe. With time, as the crystalline domains grow, the intensity of the diffraction peaks also grows, and the (02) $q_{\|}=1.600 \AA^{-1}$ peak becomes observable. This trend of increasing intensity was indeed observed on the (11) peak with a half-intensity lifetime of roughly $1.5 \mathrm{~h}$.

We focus now on the characteristics of the two peaks in the equilibrium phase observed $\sim 5 \mathrm{~h}$ after film deposition. The indexing of these peaks as (11) and (02) implies an intensity ratio of $I(1.384) \approx 2 I(1.600)$, as indeed observed in Figure 4. The molecular area, $5.56 \AA \times 7.85 / 2 \AA=21.8 \AA^{2} /$ molecule, is very close to that found for SAMs of MMB on Au. ${ }^{2}$ The 5.56 $\AA$ length of lattice vector $\mathbf{a}$ is almost commensurate with the $3.18 \AA$ lateral interatomic distance of the mercury atoms at the surface: $5.56 / 3.18 \approx \sqrt{ } 3$. A similar correspondence with the $\mathrm{Hg}-\mathrm{Hg}$ atomic distance at the surface was also found for alkylthiols on mercury, where the $\mathrm{Hg}$-commensurate length of a remains constant upon increasing the length of the alkyl chain, and hence also the length of the second lattice vector $\mathbf{b} .{ }^{20}$ The similarity between the structures of alkyl-thiols and the biphenylthiols on mercury highlights the dominant influence of the thiol group's strong affinity for the mercury in determining the structure. The main difference between the structures is that the thiol headgroups of the alkyl-thiols form a noncentered unit cell, indicated by the appearance of odd $-(h+k)$ GID peaks, due to the binding of two headgroups in each unit cell to a single mercury atom. ${ }^{20}$ By contrast, the absence of such odd $(h+k)$ peaks here indicates that no such bonds occur for MMB.

The fwhm of the GID peaks is $\Delta q_{\|} \approx 0.018 \AA^{-1}$ which is close to the resolution limit $\Delta q_{\| \text {res }}=0.017 \AA^{-1}$. This yields, through the Debye - Scherrer formula $\xi=2 \pi /\left(\Delta q_{\|}^{2}-\Delta q_{\| \text {res }}^{2}\right)^{1 / 2}$, a large crystalline coherence length ${ }^{1} \xi>1000 \AA$. This is a characteristic of the herringbone-packed crystalline CS phase of Langmuir films on mercury ${ }^{19,20}$ and on water, ${ }^{1}$ while hexagonally packed rotator phases are found to have shorter coherence lengths, on the order of a few hundred angstroms only, 1,17,19,20 similar to that found below for FMMB. This similarity implies a herringbone-like molecular packing for MMB, rather than a rotator one. This conclusion is further supported by the observation of an anisotropic expansion coefficient for MMB, another characteristic of the CS herringbone packing in Langmuir films, as discussed below, and the rather small area per molecule at this phase, which would hinder a free rotation of the molecules.

The Bragg rods measured at the two GID positions are shown in panels $\mathrm{b}$ and $\mathrm{c}$ of Figure 4, along with their fits to a rigidmolecule model having a uniform density and cross section along the molecular axis. ${ }^{1,17,30}$ The intensity distribution in the BR along $q_{\mathrm{z}}$ is determined by the product of the molecular form factor and the structure factor of the $2 \mathrm{D}$ crystal. The Bragg rod is characterized by a sharp surface-enhancement peak at $q_{\mathrm{z}}=$ $q_{\mathrm{c}}$ (known as the "Vineyard peak"37) on top of a broader distribution, the width of which is inversely proportional to the projection of the length of the molecule onto the surface normal. The $q_{\mathrm{z}}$ position of the maximum of the broad distribution depends on the tilt of the molecules and its direction. For the MMB data shown in Figure 4, the BR peak positions are $q_{\mathrm{z}}{ }^{0}=$ $0.28 \AA^{-1}$ for the $q_{\|}=1.384 \AA^{-1}$ GID peak and $q_{\mathrm{z}}{ }^{0}=0 \AA^{-1}$ for the $q_{\|}=1.600 \AA^{-1}$ GID peak. This combination indicates ${ }^{1}$ a molecular tilt of $\theta=\tan ^{-1}\left\{q_{\mathrm{z}}{ }^{0}(11) /\left[q_{\|} \|^{2}(11)-q_{\|^{2}}(02) / 4\right]\right\}^{1 / 2}$ $=13.9^{\circ}$ from the surface normal in the direction of $\mathbf{a}$, which is the nearest-neighbor $(\mathrm{NN})$ direction. A fit of the measured BRs by a widely used model, ${ }^{17,19,30}$ shown in lines in panels $\mathrm{b}$ and 

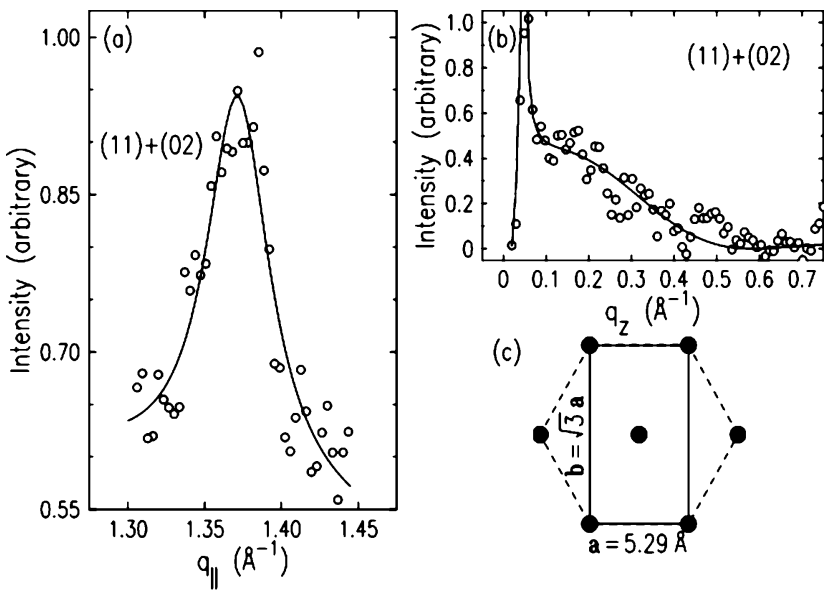

Figure 5. Measured (O) and model-fitted (-) GID (a) and BR (b) for the standing-up phase of the FMMB at room temperature. (c) Hexagonal in-plane order as represented by a centered rectangular unit cell of 5.29 $\AA \times 5.29 \sqrt{ } 3 \AA=5.29 \AA \times 9.16 \AA$.

TABLE 2: GID Peak Positions at the Indicated Temperatures for Hexacosanol, Tetracosanoic Acid, and MMB

\begin{tabular}{ccccccccc}
\hline & \multicolumn{2}{c}{$\mathrm{C}_{26} \mathrm{OH}$ alcohol } & & \multicolumn{2}{c}{$\mathrm{C}_{24} \mathrm{OOH}$ fatty acid } & & \multicolumn{2}{c}{$\mathrm{MMB}$} \\
\cline { 2 - 3 }$\left({ }^{\circ} \mathrm{C}\right)$ & $(11)$ & $(02)$ & & $(11)$ & $(02)$ & & $(11)$ & $(02)$ \\
\hline 10 & 1.515 & 1.689 & & 1.516 & 1.712 & & 1.386 & 1.606 \\
25 & 1.511 & 1.676 & & 1.512 & 1.688 & & 1.384 & 1.600
\end{tabular}

c of Figure 4, agrees well with the measured BRs and supports the conclusions drawn from the peak positions.

$F M M B$. The high-coverage, standing-up phase of FMMB, at a nominal $A=25.7 \AA^{2} /$ molecule, exhibits only a single GID peak at $q_{\|}=1.372 \AA^{-1}$, shown in Figure 5. This indicates a hexagonal phase, with a corresponding centered rectangular unit cell with dimensions of $5.29 \AA \times 5.29 \sqrt{ } 3 \AA$ and $A_{\mathrm{x}}=24.2$ $\AA^{2} /$ molecule. The intensity of this peak is only $\leq 10 \%$ of that of the (11) peak of MMB, shown in Figure 4. Moreover, the FMMB peak is much broader than the nearly resolution limited peaks of MMB. Its fwhm width, $0.050 \AA^{-1}$, corresponds to a coherence length $\xi$ of only $\sim 110 \AA$, as compared to the $\xi$ of $>1000 \AA$ of MMB. The 10 -fold lower $\xi$ and $10 \%$ larger $A_{\mathrm{x}}$ lead to the conclusion that this phase is a rotator phase, in contrast with MMB, where the corresponding phase is a herringbone crystal.

The measured BR of FMMB at this coverage, shown in Figure $5 \mathrm{~b}$, peaks at $q_{\mathrm{z}}^{0}=0 \AA^{-1}$, indicating that the molecules are oriented along the surface normal and are untilted. The BR's fwhm and the model fit, shown in a line in Figure 5b, yield a surface-normal projected length of $14 \AA$ for the ordered molecules. This value coincides, within the experimental and fit errors, with the value of $14.1 \AA$ derived from the XR measurements and listed in Table 1, and the bond-lengths calculated value of $14.2 \AA$. These values support therefore the conclusion that the FMMB molecules are untilted. This is again in contrast with the tilted phase found for $\mathrm{MMB}$ at the corresponding nominal coverage $A$, as discussed above.

Temperature Dependence. A few temperature-dependent GID measurements were carried out for stable MMB films, i.e., more than $5 \mathrm{~h}$ after film deposition. These were done in the standing-up phase at a nominal $A=30 \AA^{2} /$ molecule. The peak positions that were obtained are summarized in Table 2, along with similar results obtained for two other Langmuir monolayers, tetracosanoic acid $\left(\mathrm{C}_{23} \mathrm{H}_{47} \mathrm{COOH}\right)^{17}$ and hexacosanol $\left(\mathrm{C}_{25} \mathrm{H}_{53}-\right.$ $\mathrm{COH})^{19}$ on mercury, in their standing-up phase. These two were selected since they exhibit in this temperature range a $2 \mathrm{D}$ crystalline herringbone $C S$ phase,${ }^{1}$ like that argued above to exist for the standing-up phase of MMB.

Table 2 shows that the expansion coefficients of all three compounds are anisotropic. The length of the lattice vector a hardly changes with temperature, possibly because of its commensuration with the $\mathrm{Hg}-\mathrm{Hg}$ surface distance mentioned above. By contrast, the length of the lattice vector $\mathbf{b}$ varies with temperature, yielding coefficients of expansion of $(\mathrm{d} b / \mathrm{d} T) / b=$ $5 \times 10^{-4}, 9.5 \times 10^{-4}$, and $2.5 \times 10^{-4} \mathrm{~K}^{-1}$ for the alcohol, the fatty acid, and MMB, respectively. The anisotropic expansion coefficient is a signature of the crystalline herringbone phase, while the rotator phases, e.g., tetradecanoic acid on mercury in the same temperature range, ${ }^{17}$ exhibit isotropic expansion coefficients. The observation of anisotropy for MMB further supports the suggestion above that the standing-up phase of MMB is a crystalline herringbone $C S$ phase. ${ }^{1}$ It is interesting to note in Table 2 that the expansion coefficient of the MMB Langmuir film on $\mathrm{Hg}$ is 2-4-fold smaller than those of Langmuir films of the linear chain molecules on $\mathrm{Hg}$. This indicates an intermolecular attraction of the biphenyl molecules which is stronger than that of the alkyl chains, resulting in an increased stiffness. The increased stiffness and stability of the MMB monolayer as compared to those of alkyl-thiols are also reflected in the significantly higher collapse pressure of the Langmuir film of MMB as compared to that of the chain molecules, and in the higher thermal stability of the MMB monolayer on $\mathrm{Au}$, the melting temperature of which is $\sim 60{ }^{\circ} \mathrm{C}$ higher than that of a Langmuir film of an equal-length alkylthiol on the same substrate. ${ }^{2}$

\section{Discussion}

Two important questions arise from the results presented above. (a) Why are the structures of the high-coverage phases of the apparently very similar MMB and FMMB molecules so different? (b) How do the results for Langmuir films of MMB and FMMB on liquid mercury compare with previous results for self-assembled monolayers of the same molecules on solid $\mathrm{Au}$ on one hand, and with those of Langmuir films of alkylthiols on liquid mercury on the other hand, and what are the molecular-level origins of the differences? We now discuss these questions in turn.

The only difference in structure between MMB and FMMB is the replacement of the methyl's hydrogens with fluorines. This apparently small change results, however, in a significant increase in the size of the terminal methyl group. Kim et al. ${ }^{38}$ calculate for the terminal $\mathrm{CH}_{3}$ moiety of an alkyl-thiol chain a cross section of $A_{\mathrm{CH}_{3}}=17.6 \AA^{2}$. Replacing this terminal group with a $\mathrm{CF}_{3}$ group increases the cross section to $A_{\mathrm{CF}_{3}}=25.4 \AA^{2}$. $A_{\mathrm{CH}_{3}}$ is much smaller than the area per molecule favored by the MMB molecule, $A_{\mathrm{x}}=21.84 \AA^{2} /$ molecule, obtained above from the X-ray GID measurements, and hence, the terminal $\mathrm{CH}_{3}$ does not impose packing constraints on the structure of MMB. By contrast, $A_{\mathrm{CF}_{3}}$ is $15 \%$ larger than the molecular area favored by the biphenyl moiety of the molecule, and hence imposes severe packing constraints on the in-plane structure. These constraints result in the molecular area of FMMB being $A_{\mathrm{x}}=$ $24.2 \AA^{2} /$ molecule, i.e., $11 \%$ larger than that of MMB. The packing frustrations due to the mismatch between the molecular areas preferred by the perfluorinated methyl and the biphenyl backbone result in the crystalline coherence length of FMMB being 10-fold shorter than that of MMB, as discussed above. As the close packing of the roughly spherical $\mathrm{CF}_{3}$ groups now dominates the in-plane order, a hexagonal phase is obtained for FMMB, rather than the rectangular packing favored by the 
platelike biphenyl backbone, which dominates the structure of the corresponding phase of MMB. We also note that the large electronegativity of the fluorine atoms should induce in the upper end of the FMMB molecule a significant dipole moment, which does not exist in the $\mathrm{CH}_{3}$-terminated $\mathrm{MMB}$, similar to the dipole moment found for $\mathrm{CF}_{3}$-terminated alkyl-thiols on $\mathrm{Au} .^{23,39}$ The repulsion between the parallel-aligned dipoles of adjacent molecules may also contribute ${ }^{40}$ to the packing frustration which reduced the crystallinity of the standing-up phase of FMMB.

$\mathrm{X}$-ray measurements ${ }^{2}$ on the lying-down phase of MMB on $\mathrm{Au}(111)$ reveal a striped phase having a long-range in-plane crystalline order with a distorted rectangular unit cell. A recent detailed STM study ${ }^{32}$ at this coverage range resolved two different striped phases, with slightly different long-range ordered structures. On mercury, however, no GID peaks are observed for the corresponding phase of lying-down molecules, indicating that the coherence length of any order that may exist within the monolayer cannot exceed very few tens of angstroms, as for a liquid or an amorphous solid. In the absence of GID peaks, the only clue about the organization of the molecules at the surface in the lying-down phase is the exclusion area derived from the Vollmer equation fit to the fast increasing part of the isotherms, which yields $A_{0}=63.5 \AA^{2} /$ molecule, as discussed above. Comparing this to the GID results of the striped phase of MMB on $\mathrm{Au}(111)$ which yields an area per molecule of 57.7 $\AA^{2} /$ molecule leads to the conclusion that the lying-down phase of MMB and FMMB on mercury at $A \approx 65$ (MMB) and 75 $\AA^{2} /$ molecule (FMMB) is such that the molecular axis is surfaceparallel, with one phenyl ring lying flat on the mercury surface. However, the fact that the XR measurements yield a $4.9 \AA$ layer thickness, larger than the $3.3 \AA$ thickness of the ring, indicates that the second phenyl ring is rotated about the molecular axis; i.e., the dihedral angle between the planes of the two rings is non-zero, as indeed found for the striped phase of MMB on $\mathrm{Au}$ as well. Moreover, on Au the molecular axis of MMB was argued to be not fully parallel to the surface, but tilted up from it by a small angle, presumably by the need to make room for the lower section of the dihedrally rotated ring but also because of the strong attraction exerted on the terminal thiol group by the $\mathrm{Au}$ atoms of the surface. .,32 $^{2}$ Such a tilt is likely to be present here as well for the very same reasons. If this is indeed the case, the XR-observed layer thickness would be a combined result of the up tilt of the molecule from the surface and the non-zero dihedral rotation angle of the rings. For a given layer thickness, the larger is the former, the smaller is the later, and vice versa. More definite conclusions about these details of the structure could have been inferred only from accurate GID patterns, which, however, do not exist due to the lack of longrange order. The lying-down phases of alkyl-thiols on mercury also lack long-range in-plane order for all carbon numbers investigated $^{20}$ ( $\left.n=9-22\right)$. This, again, is in contrast with alkanethiols on $\mathrm{Au}(111)$ which show long-range ordered striped phases $^{41}$ akin to those observed for MMB on Au. ${ }^{2,32}$ The discussion leads, therefore, to the not very surprising conclusion that the subphase's structure, or lack thereof, plays a major role in determining the structure of the lying-down monolayer: for the long-range ordered Au subphase, a commensurate order (at least in one direction) is epitaxially induced in the organic overlayer, while for the disordered (or short-range ordered) liquid $\mathrm{Hg}$ interface, a similarly disordered (or short-range ordered) layer is obtained.

Previous X-ray measurements on the standing-up phase of $\mathrm{MMB}$ on $\mathrm{Au}^{2}$ reveal a hexagonal order with a rather poor crystalline coherence length and a unit cell of $4.99 \AA \times 8.65$
$\AA$, yielding an area per molecule of $A_{\mathrm{x}}=21.6 \AA^{2} /$ molecule. The molecules were found to tilt by $<19^{\circ}$ from the surface normal, but the accurate magnitude and azimuthal direction of the tilt could not be determined. A recent STM study reveals that a $2 \times 1$ superlattice is first formed $\sim 3 \mathrm{~h}$ after immersion of the Au substrates in the MMB solution. This phase converts to the hexagonal phase at longer immersion times. ${ }^{32}$ This structure is in marked contrast with the equilibrium phase of MMB on mercury, discussed above, which has a rectangular cell structure of $5.56 \AA \times 7.84 \AA$, and thus a (similar) area per molecule of $21.84 \AA^{2} /$ molecule. The $A_{\mathrm{x}}$ values of MMB on both $\mathrm{Au}$ and $\mathrm{Hg}$ are close to the value of $22.1 \AA^{2} /$ molecule derived from the $3 \mathrm{D}$ crystal structure of biphenyl, which has a unit cell of $5.51 \AA \times 8.04 \AA$ in the $(x, y)$ plane. This indicates that the biphenyl core of the MMB dominates the structure of the standing-up monolayer phase. Note, however, that although the area per molecule is similar, the unit cell dimensions are not. Compared to the dimensions of the biphenyl $(x, y)$ unit cell, that of MMB on mercury deviates by only $0.9 \% \times 2.5 \%$, while that on $\mathrm{Au}$ deviates by a much larger amount $(9.5 \% \times 7.5 \%)$. The opposite signs of the deviations in the two lattice-vector directions keep the area almost unchanged.

The different behavior of $\mathrm{MMB}$ on $\mathrm{Hg}$ and $\mathrm{Au}$ can be attributed, again, to the structure of the subphase. For MMB on $\mathrm{Au}$, the larger distortions of the biphenyl's native unit cell are imposed by strong epitaxy to the crystalline structure of $\mathrm{Au}$. With these cell dimensions, all S headgroups of MMB indeed reside at the three-atom hollows of the hexagonally packed $\mathrm{Au}(111)$ surface. However, the packing frustration resulting from the unit cell dimensions favored by the biphenyl core and those favored by the $\mathrm{S}$ headgroups result in the poor crystallinity, i.e., rather short crystalline coherence length, observed in both X-ray ${ }^{2}$ and $\mathrm{STM}^{32}$ studies of MMB on Au. On liquid mercury, where the $\mathrm{S}-\mathrm{Hg}$ bond strength, $>200 \mathrm{~kJ} /$ mol, is similar to that of the $\mathrm{S}-\mathrm{Au}$ bond, ${ }^{36}$ but no long-range subphase order exists, no such constraints are imposed, and the deviations from the native structure are much smaller. However, even here a very weak epitaxy, albeit one-dimensional only, is observed: the lattice vector length $|\mathbf{a}|=5.56 \AA$ coincides almost exactly with $\sqrt{ } 3 \times 3.18 \AA$, where $3.18 \AA$ is the $\mathrm{Hg}-\mathrm{Hg}$ interatomic distance at the surface at room temperature. ${ }^{20} \mathrm{~A}$ similar one-dimensional weak epitaxy to the $\mathrm{Hg}-\mathrm{Hg}$ interatomic distance at the surface is found for the standing-up monolayer phase of alkyl-thiols on mercury, where, again, a lattice vector length of $\sqrt{3} \times 3.18 \AA$ is found. ${ }^{20}$

Another type of deviation from the native structure occurs for FMMB. Here the bulky end group results in a hexagonal unit cell, $5.29 \AA \times 5.29 \sqrt{3} \AA$, much larger than that favored by the biphenyl core. In line with our conclusions above, the packing frustration leads here, as for $\mathrm{MMB}$ on $\mathrm{Au}$, to a coherence length considerably shorter than that of $\mathrm{MMB}$ on $\mathrm{Hg}$, to a hexagonal, rather than a rectangular, packing, and to a loss of even the weak one-dimensional epitaxy to the surface interatomic $\mathrm{Hg}$ distance observed for $\mathrm{MMB}$ on $\mathrm{Hg}$. A structural study of FMMB on Au, not currently available, would help in further elucidating the interplay between the molecular cross section and the subphase epitaxy in determining the structure of solid- and liquid-supported organic monolayers.

Finally, it is interesting to compare the structure of the standing-up phase of MMB on mercury with the corresponding phase of alkyl-thiols. While the structure of $\mathrm{CH}_{3}\left(\mathrm{CH}_{2}\right)_{9} \mathrm{SH}$ (denote $\mathrm{C}_{10} \mathrm{~S}$ ) on mercury, the length of which is roughly equal to that of $\mathrm{MMB},{ }^{2}$ has not been studied by X-rays, the not-muchlonger $\mathrm{C}_{14} \mathrm{~S}$ was studied in detail. ${ }^{20}$ The standing-up phase of 
alkyl-thiols has a rectangular unit cell with a fixed $26 \pm 2^{\circ}$ tilt from the surface normal, independent of $n$. The molecular area measured ${ }^{20}$ for $\mathrm{C}_{14} \mathrm{~S}$ in the plane perpendicular to the chains is $A_{\perp}=21.3 \AA^{2} /$ molecule. Taking into consideration the $\mathrm{NN}$ directed $13.9^{\circ}$ molecular tilt, the corresponding $\mathrm{A}_{\perp}$ for $\mathrm{MMB}$ is $21.84 \times \cos (13.9)=21.2 \AA^{2} /$ molecule, rather close to that of $\mathrm{C}_{14} \mathrm{~S}$. However, the much larger tilt, $\sim 27^{\circ}$, of the alkyl-thiols renders the surface-parallel area per molecule of $\mathrm{C}_{14} \mathrm{~S}$ significantly larger $\left(\sim 23.5 \AA^{2} /\right.$ molecule $)$. This difference in the surface-parallel area per molecule, and hence in the spacing of the $\mathrm{S}$ headgroups, has important consequences. For $\mathrm{C}_{18} \mathrm{~S}$, the appearance of odd $-(h+k)$ peaks in the GID pattern indicated a different packing for the chains and for the headgroups. ${ }^{20}$ While the former pack in a centered rectangular cell, the latter pack in a noncentered cell. This was explained by the formation of one $\mathrm{S}-\mathrm{Hg}-\mathrm{S}$ bond per unit cell, and the existence of longrange orientational order for these bonds. Reducing the nominal molecular area to $19 \AA^{2} /$ molecule yields a transition to a hexagonal phase, where the bond orientational order and the noncentered cell of the headgroups are not observed. ${ }^{42}$ The absence of odd $-(h+k)$ GID peaks for MMB on mercury in this phase implies the absence of $\mathrm{S}-\mathrm{Hg}-\mathrm{S}$ bonding, presumably because the smaller unit cell is unable to accommodate the somewhat longer $\mathrm{S}-\mathrm{Hg}-\mathrm{S}$ contacts required for the bond. It is also possible that these bonds do exist but possess a liquidlike, short-range order. This results in broad, shallow peaks not distinguishable in a GID scan above the background. The increased rigidity of the biphenyl core as compared to the alkyl chain and the consequent lack of the ability to accommodate two different, albeit very close, structures by different parts of the same molecule may also inhibit the formation of $\mathrm{S}-\mathrm{Hg}-\mathrm{S}$ bonds with long-range orientational order.

\section{Conclusions}

We present here a detailed surface tensiometry and X-ray study of the structure of MMB and FMMB on mercury. The results were compared to those of the same molecules both on a solid $\mathrm{Au}$ substrate and on alkanethiols on both $\mathrm{Au}$ and mercury. The results discussed above demonstrate the dominance of two factors, namely, the structure, or lack thereof, of the subphase and the cross section and rigidity of the backbone, in determining the structure of the various phases of organic films on both liquid and solid surfaces, formed either by selfassembly or as Langmuir films. In addition, the replacement of the hydrophobic interaction of a water subphase with an organic Langmuir film with a strong attraction of the molecules to the liquid metal surface results in lying-down phases for both MMB and FMMB, phases not observed in Langmuir films of organic molecules on water. ${ }^{1}$

This study will be extended to other ring compounds, like the acene and their derivatives, which show great promise for single- or few-molecule device applications in molecular electronics.

Acknowledgment. Support to M.D. by the U.S.-Israeli Binational Science Foundation, to P.S.P. by the NSF through Grant NSF-DMR-0124936 and by DOE Grant DE-FG02-88ER45379, and to A.U. by the NSF through the MRSEC for Polymers at Engineered Surfaces, when he was at the Polytechnic University, is gratefully acknowledged. Beamtime at Beamline X22B, NSLS, is also acknowledged gratefully. Brookhaven National Laboratory is supported by the U.S. Department of Energy under Contract DE-AC02-76CH00016.

\section{References and Notes}

(1) Kaganer, V. M.; Mohwald, H.; Dutta, P. Rev. Mod. Phys. 1999, 71,779 .

(2) Leung, T. Y. B.; Schwartz, P.; Scoles, G.; Schreiber, F.; Ulman, A. Surf. Sci. 2000, 458,34

(3) Jin, Q.; Rodriguez, J. A.; Li, C. Z.; Darici, Y.; Tao, N. J. Surf. Sci. 1999, 425, 101 .

(4) Bandyopadhyay, K.; Vijayamohanan, K.; Shekhawat, G. S.; Gupta, R. P. J. Electroanal. Chem. 1998, 447, 11.

(5) Mantooth, B. A.; Weiss, P. S. Proc. IEEE 2003, 91, 1785. Jortner, J., Ratner, M. Eds. Molecular Electronics; Blackwell: Oxford, U.K., 1997.

(6) Ulman, A. An Introduction to Ultrathin Organic Films; Academic: Boston, 1991. Schreiber, F. Prog. Surf. Sci. 2000, 65, 151.; J. Phys.: Condens. Matter 2004, 16, R881. Ulman, A. Thin Films: SelfAssembled Monolayers of Thiols; Academic: San Diego, 1998.

(7) Ulman, A. Acc. Chem. Res. 2001, 34, 855.

(8) Schmelmer, U.; Jordan, R.; Geyer, W.; Eck, W.; Gölzhäuser, A.; Grunze, M.; Ulman, A. Angew. Chem., Int. Ed. 2003, 42, 559.

(9) Zaccaro, J.; Kang, J. F.; Ulman, A.; Myerson, A. S. Langmuir 2000 , 16,3791

(10) Jordan, R.; Ulman, A.; Kang, J. F.; Rafailovich, M.; Sokolov, J. J. Am. Chem. Soc. 1999, 121, 1016.

(11) Yu, H. Z.; Xia, N.; Zhang, J.; Liu, Z. F. J. Electroanal. Chem. 1998, 448, 119 .

(12) Rampi, M. A.; Whitesides, G. M. Chem. Phys. 2002, 281, 373

(13) Selzer, Y.; Salomon, A.; Cahen, D. J. Am. Chem. Soc. 2002, 124 2886.

(14) Grayer Wolf, S.; Leiserowitz, L.; Lahav, M.; Deutsch, M.; Kjaer, K.; Als-Nielsen, J. Nature 1987, 328, 63. Grayer Wolf, S.; Deutsch, M.; Landau, E. M.; Lahav, M.; Leiserowitz, L.; Kjaer, K.; Als-Nielsen, J. Science 1988, 242, 1286. Kjaer, K.; Als-Nielsen, J.; Helm, C. A.; Laxhuber, L. A.; Möhwald, H. Phys. Rev. Lett. 1987, 58, 2224. Dutta, P.; Peng, J. B.; Lin, B.; Ketterson, J. B.; Prakash, M. Phys. Rev. Lett. 1987, 58, 2228.

(15) Nuzzo, R. G.; Allara, D. J. J. Am. Chem. Soc. 1983, 105, 4481.

(16) Sagiv, J. J. Am. Chem. Soc. 1980, 102, 92.

(17) Kraack, H.; Ocko, B. M.; Pershan, P. S.; Deutsch, M. Science 2002. 298, 1404. Kraack, H.; Deutsch, M.; Ocko, B. M.; Pershan, P. S. Nucl. Instrum. Methods Phys. Res., Sect. B 2003, 200, 363. Kraack, H.; Ocko, B. M.; Pershan, P. S.; Sloutskin, E.; Tamam, L.; Deutsch, M. Langmuir 2004, 20,5375 .

(18) Kraack, H.; Ocko, B. M.; Pershan, P. S.; Sloutskin, E.; Deutsch, M. J. Chem. Phys. 2003, 119, 10339.

(19) Kraack, H.; Ocko, B. M.; Pershan, P. S.; Sloutskin, E.; Tamam, L.; Deutsch, M. Langmuir 2004, 20, 5386.

(20) Ocko, B. M.; Kraack, H.; Pershan, P. S.; Sloutskin, E.; Tamam, L.; Deutsch, M. Phys. Rev. Lett. 2005, 94, 017802.

(21) Ishida, T.; Mizutani, W.; Choi, N.; Akiba, U.; Fujihira, M. Tokumoto, H. J. Phys. Chem. B 2000, 104, 11680.

(22) Wang, W.; Zhai, J.; Bai, F. Chem. Phys. Lett. 2002, 366, 165.

(23) Pflaum, J.; Bracco, G.; Schreiber, F.; Colorado, R., Jr.; Shmakova,

O. E.; Lee, T. R.; Scoles, G.; Kahn, A. Surf. Sci. 2002, 498, 89.

(24) Ulman, A.; Kang, F. K.; Jordan, R.; Kurth, D. G. Langmuir 1999 15,5555 .

(25) Magnussen, O. M.; Ocko, B. M.; Regan, M. J.; Penanen, M. J.; Deutsch, M.; Pershan, P. S. Phys. Rev. Lett. 1995, 74, 4444. DiMasi, E.; Tostmann, H.; Ocko, B. M.; Parshan, P. S.; Deutsch, M. Phys. Rev. B 1998, $58, \mathrm{R} 13419$.

(26) Magnussen, O. M.; Ocko, B. M.; Deutsch, M.; Regan, M. J.; Pershan, P. S.; Abernathy, D.; Grübel, G.; Legrand, J. F. Nature 1996, 384, 250.

(27) Gaines, G. L. Insoluble Monolayers at Liqid Gas Interface; Wiley: New York, 1966.

(28) Kang, J. F.; Ulman, A.; Liao, S.; Jordan, R.; Yang, G.; Liu, G.-Y. Langmuir 2000, 16, 95.

(29) Smith, T. Adv. Colloid Interface Sci. 1972, 3, 161.

(30) Lekner, J. Theory of Reflaction; Martinus Nijhoff: Dordrecht, The Netherlands, 1987. Abeles, F. Ann. Phys. 1950, 5, 596.

(31) Deutsch, M.; Ocko, B. M. In Encyclopedia of Applied Physics Trigg, G. L., Ed.; VCH: New York, 1998; Vol. 23, p 479. Als-Nielsen, J.; McMorrow, D. Elements of Modern X-ray Physics; Wiley: New York, 2001.

(32) Azzam, W.; Fuxon, C.; Birkner, A.; Rong, H. T.; Buck, M.; Wöll, Ch. Langmuir 2003, 19, 4958.

(33) Ocko, B. M.; Wu, X. Z.; Sirota, E. B.; Sinha, S. K.; Gang, O.; Deutsch, M. Phys. Rev. E 1997, 55, 3164

(34) Watanabe, Y. Acta Crystallogr. 1977, B33, 3566. Cundari, T. R.; Yoshikawa, A. J. Comput. Chem. 1998, 19, 902. This bond length depends somewhat on the particular molecule where it occurs.

(35) Szczesniak, M. M.; Chalasinski, G.; Cybulski, S. M.; Scheiner, S.

J. Chem. Phys. 1990, 93, 4234.

(36) CRC Handbook of Chemistry and Physics, 66th ed.; Weast, R. C., Astle, M. J., Beyer, W. H., Eds.; CRC Press: Boca Raton, FL, 1986.

(37) Vineyard, G. H. Phys. Rev. B 1982, 26, 4146. 
(38) Kim, H. I.; Koini, T.; Randall, T. R.; Perry, S. S. Lanngmuir 1997, 13,7192 .

(39) Graupe, M.; Takemaga, M.; Koini, T.; Colorado, R., Jr.; Lee, T. R. J. Am. Chem. Soc. 1999, 121, 3222. Houssiau, L.; et al. J. Chem. Phys. 1998, 109, 9134.

(40) Kang, J. F.; Kurth, D. G.; Ulman, A.; Jordan, R. Langmuir 1999, 15,5555 .
(41) Fenter, P.; Eberhardt, A.; Eisenberger, P. Science 1994, 266, 1216

(42) Upon compression to $19 \AA^{2} /$ molecule at room temperature, this phase appears to be in coexistence with the lower-coverage, $27^{\circ}$-tilted phase The pure untilted hexagonal phase emerges only upon cooling to below 10 ${ }^{\circ} \mathrm{C}$. However, the pure hexagonal phase persists upon reheating to room temperature. 